\title{
Diagnostic and Therapeutic Challenges Associated with the Rare Cardiac Metastasis Complicating Rectal Adenocarcinoma
}

\author{
Mohammad Alrehaili ${ }^{a}$ Emad Tashkandi ${ }^{a}$ b \\ aDepartment of Medical Oncology, Oncology Center, King Abdullah Medical City, Makkah, \\ Saudi Arabia; ${ }^{b}$ College of Medicine, Umm Al-Qura University, Makkah, Saudi Arabia
}

\author{
Keywords \\ Cardiac metastasis · Rectal cancer · Adenocarcinoma · Cardiac MRI, Transthoracic \\ echocardiography
}

\section{Abstract}

Colorectal cancer (CRC) is one of the most common malignancies in the world. It typically metastasizes to the lymph nodes, liver or lungs. Cardiac involvement is considered the least likely metastatic complication of malignant tumors including CRC. We report a rare case of metastatic rectal cancer to the liver, ovaries, lungs and peritoneum that presented initially with a 1-week history of rectal bleeding. Her hospital course was complicated by progressive dyspnea and palpitations secondary to right atrial metastasis based on imaging studies including cardiac MRI with contrast. The patient was not fit for chemotherapy or any surgical intervention given her poor prognosis and functional status in the setting of advanced stage of her disease. After discussion with the patient and her family, a decision was made to change her code status to DNR (do not resuscitate) and focus on palliative treatment of her disease. She expired about 2 weeks following her discharge date. Based on this case report, we recommend a high index of suspicion for cardiac metastasis when dealing with cardiac or respiratory complaints in cases of CRC that need careful evaluation with echocardiography and MRI. 


\section{Introduction}

Cardiac metastases are considered to be a rare complication of primary malignant tumors. Its exact incidence reported in the literature is highly variable. It is usually asymptomatic, but could present with nonspecific symptoms that lead to diagnostic and therapeutic challenges. The tumors that show the highest rate of heart metastasis include pleural mesothelioma, melanoma and lung cancer [1]. Less metastatic potential was observed in some tumors arising from the ovaries, stomach, kidney and pancreas. However, the least likely malignancies to metastasize to the heart include colorectal and prostatic cancers [1,2]. Here, we report a rare case of rectal adenocarcinoma that presented initially with rectal bleeding and was found later having right atrial metastasis that was confirmed by cardiac MRI with contrast.

\section{Case Report}

A 54-year-old female with a past history of left lower extremity deep vein thrombosis on Apixaban was transferred to our hospital due to a 1-week complaint of rectal bleeding. Associated symptoms included moderate to severe central abdominal pain for 1 month. Upon our evaluation, the patient looked in mild distress with poor functional status. Her vital signs were normal, but the abdomen was diffusely tender on palpation and remarkable for shifting dullness. The basic laboratory workup showed microcytic anemia with a hemoglobin level at $9.6 \mathrm{~g} / \mathrm{dL}$ and mild acute kidney injury with BUN at $20 \mathrm{mg} / \mathrm{dL}$ and creatinine of $1.9 \mathrm{mg} / \mathrm{dL}$. Cancer antigen 125 and carcinoembryonic antigen were elevated at 147.8 and 2,135 ng/mL, respectively. Septic screen was unremarkable.

Colonoscopy was done which showed a polypoid rectosigmoid mass and the biopsy findings were consistent with well-differentiated adenocarcinoma in a background of tubulovillous adenoma (Fig. 1). Staging CT scans revealed moderate ascites and multiple findings including multiple variable sized liver lesions with contrast enhancement, bilateral solid ovarian masses measuring $8 \times 5 \times 7 \mathrm{~cm}$ on the left side and $5 \times 5 \times 4.6 \mathrm{~cm}$ on the other side, small basal pulmonary nodules and small omental deposits.

The hospital course was complicated by progressive respiratory distress requiring ICU transfer for monitoring. Chest X-ray and arterial blood gases did not show significant abnormalities that can explain her new complaint. EKG showed sinus tachycardia and CT angiogram was negative for pulmonary embolism. Transthoracic echocardiography revealed a large mass in the right atrium measuring $3.8 \times 3 \mathrm{~cm}$, which seems to be attached to the interatrial septum and protrudes into the right ventricle through the tricuspid valve during diastole (Fig. 2). Cardiac MRI with contrast was performed for better characterization of the mass
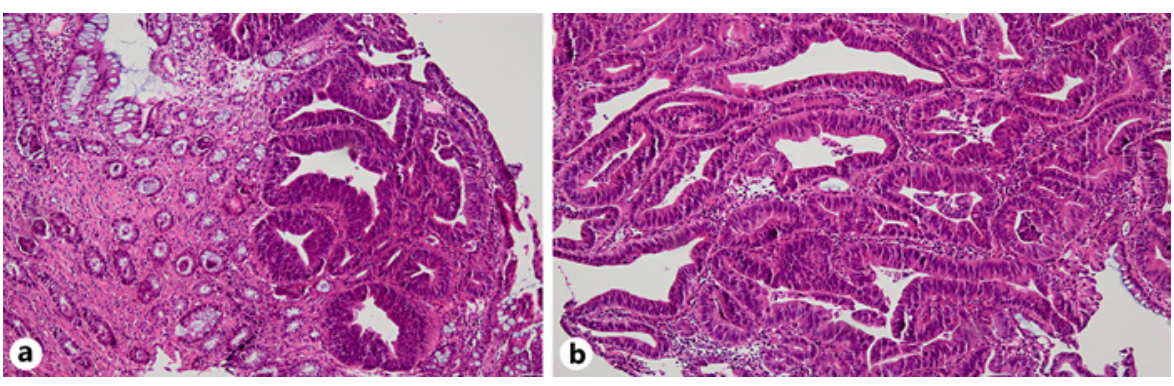

Fig. 1. Histopathology of the rectal mass showed well-differentiated adenocarcinoma (a, b). A magnified high-power field of the adenocarcinoma is also seen (b). 


\section{Case Reports in Oncology}
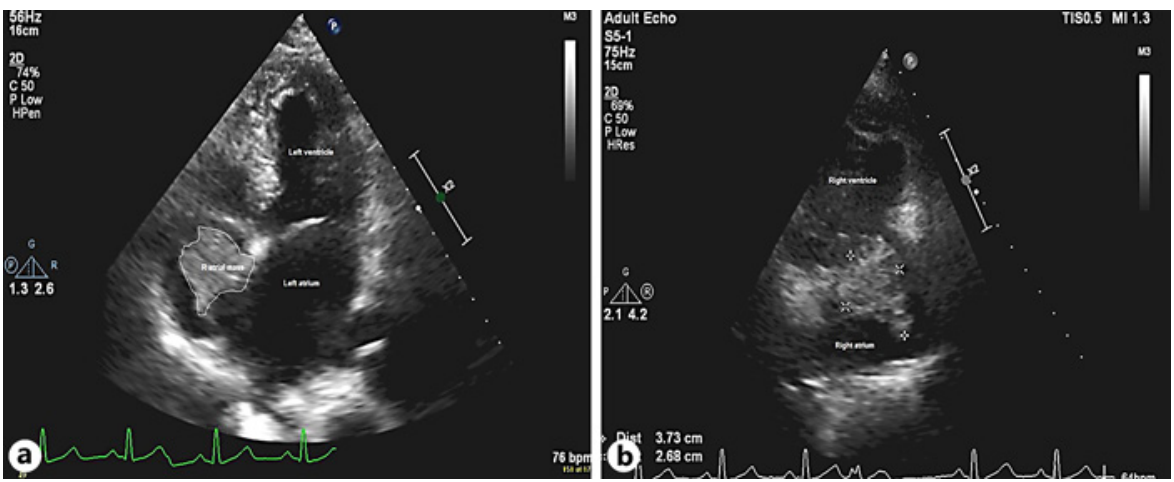

Fig. 2. Transthoracic echocardiography showed a large mass in the right atrium which seems to be attached to the interatrial septum (a). A magnified picture of the same mass measuring $3.8 \times 3 \mathrm{~cm}$ is also shown (b).
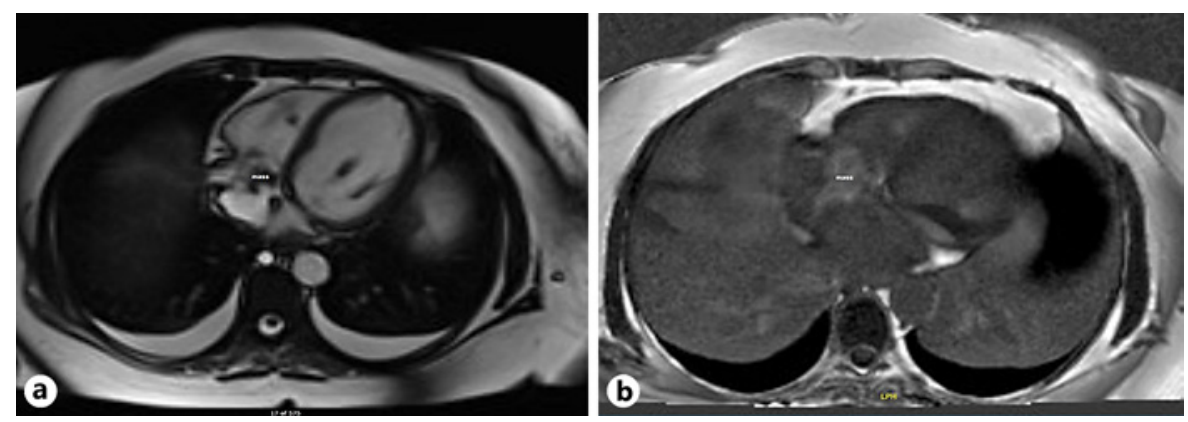

Fig. 3. Cardiac MRI showed an irregular-shaped right atrial mass (a) that revealed marked enhancement on contrast-enhanced imaging (b).

(Fig. 3). It showed a heterogenous mass of irregular and multilobed shape with contrast enhancement that is consistent with atrial metastasis.

Throughout the patient hospitalization, the anticoagulation treatment had been stopped, which led to resolution of rectal bleeding. However, inferior vena cava filter was placed with no complications due to the risk of embolization. The patient was not fit for chemotherapy or any surgical intervention given her poor prognosis and functional status in the setting of advanced stage of her disease. After discussion with the patient and her family, a decision was made to change her code status to DNR (do not resuscitate) and focus on palliative treatment of her disease. Ultimately, she was discharged home and planned for home hospice before she died within 2 weeks of her date of discharge.

\section{Discussion/Conclusion}

Colorectal cancer is one of the most frequent causes of cancer-related morbidity and mortality. It typically metastasizes to the lymph nodes, liver or lungs. However, unusual sites of metastases including the heart, spleen and thyroid gland might also occur [1-5]. It was reported that cardiac involvement is considered a very rare metastatic complication from CRC. Autopsy studies have revealed that the cardiac metastatic potential was found among the lowest in patients affected by CRC with a prevalence ranging between 1.4 and $2 \%$ as opposed to bronchogenic carcinoma that is associated with a $23-31 \%$ chance of cardiac 
metastasis cancer [1,2]. Although some cases of colon cancer metastasizing to the heart were reported in the literature, very few cases of cardiac metastasis from the rectum $[6,7]$ as seen in this case were described.

Cardiac metastases usually involve the right side of the heart [1]. They present diagnostic challenges as their clinical presentation is highly variable and can be confused with primary endocardial tumors, vegatations or organized thrombi $[6,8,9]$. They can be clinically silent or present with nonspecific symptoms including dyspnea and those related to heart failure, arrhythmias and thromboembolism [6, 8, 9]. Moreover, the tumor thrombus can extend to the inferior or superior vena cava resulting in obstructive complications $[6,10]$. Initial evaluation is usually performed using a transthoracic echocardiography which, in most cases, does not differentiate the metastatic lesion from the other possible etiologies $[6,8]$. Therefore, contrast-enhanced cardiac MRI, which has the advantage of providing more details on the pericardium, myocardial walls and cardiac chambers, is highly recommended for better evaluation of the cardiac mass and reaching to the accurate diagnosis $[11,12]$. The addition of intravenous contrast is of diagnostic value that can distinguish the tumor which enhances from the vegetations and thrombi which do not $[6,8]$.

The treatment of cardiac metastasis can be very challenging to clinicians. There are some options that can be offered mainly for palliative purposes including surgery and chemotherapy depending on the clinical status of the disease and metastatic burden. However, there is no established standard approach that can be followed for treating such cases. Certain studies have shown that some survival benefits can be seen with surgical resection of the cardiac mass $[13,14]$. On the other hand, the patients should be carefully selected as the surgical option can contribute to significant morbidity and mortality, especially in elderly people and those with diffuse metastasis.

Chemotherapy might have a role in the management of cardiac metastasis although there is little evidence supporting its effectiveness [10]. Tsujii et al. [15] reported a case of metastatic colon cancer to the right ventricle that responded well to oxaliplatin-based chemotherapy plus panitumumab. They revealed a significant decrease in the cardiac tumor size and serum levels of CEA and CA19-9 after several courses of treatment. In our patient, chemotherapy option was considered not a suitable option given her very poor prognosis.

In conclusion, right atrial involvement from rectal adenocarcinoma is extremely rare. A high index of suspicion is recommended when dealing with cardiorespiratory manifestations in cases of CRC. Due to the lack of standardized approach for such cases, further studies and clinical trials are needed to establish treatment guidelines.

\section{Statement of Ethics}

This case report was approved by the IRB at King Abdullah Medical City and written informed consent for publication was obtained from the patient's son.

\section{Conflict of Interest Statement}

The authors have no conflicts of interest to declare.

\section{Funding Sources}

No funding was provided for this case report.

\section{Karger'}




\section{Case Reports in Oncology}

\begin{tabular}{l|l}
\hline Case Rep Oncol 2020;13:1441-1445 \\
\hline DOI: 10.1159/000511600 & $\begin{array}{l}\text { @ 2020 The Author(s). Published by S. Karger AG, Basel } \\
\text { www.karger.com/cro }\end{array}$ \\
\hline
\end{tabular}

Alrehaili/Tashkandi: Cardiac Metastasis Complicating Rectal Adenocarcinoma

\section{Author Contributions}

Mohammad Alrehaili wrote the paper with input from Emad Tashkandi.

\section{References}

1 Bussani R, De-Giorgio F, Abbate A, Silvestri F. Cardiac metastases. J Clin Pathol. 2007;60(1):27-34.

2 Choufani EB, Lazar HL, Hartshorn KL. Two unusual sites of colon cancer metastases and a rare thyroid lymphoma. Case 2. Chemotherapy-responsive right atrial metastasis from colon carcinoma. J Clin Oncol. 2001; 19(15):3574-5.

3 Bigot P, Goodman C, Hamy A, Teyssedou C, Arnaud JP. Isolated splenic metastasis from colorectal cancer: report of a case. J Gastrointest Surg. 2008;12(5):981-2.

4 Choi PW, Kim CN, Kim HS, Lee JM, Heo TG, Park JH, et al. Skeletal muscle metastasis from colorectal cancer: report of a case. J Korean Soc Coloproctol. 2008;24(6):492.

5 Phillips JS, Lishman S, Jani P. Colonic carcinoma metastasis to the thyroid: a case of skip metastasis. J Laryngol Otol. 2005;119(10):834-6.

6 Ayyalaa SS, Urcuyoa DM, Kannarkatta PT, Kovacsb JE, Terrignoa NJ. A Rare Case of Atrial Metastasis From a Rectal Adenocarcinoma. J Clin Med Res. 2017;9(10):886-8.

7 Koizumi J, Agematsu K, Ohkado A, Shiikawa A, Uchida T. Solitary cardiac metastasis of rectal adenocarcinoma. Jpn J Thorac Cardiovasc Surg. 2003;51(7):330-2.

8 Patel SA, Herfel BM, Nolan MA. Metastatic colon cancer involving the right atrium. Tex Heart Inst J. 2012;39(1): 79-83.

9 Choi PW, Kim CN, Chang SH, Chang WI, Kim CY, Choi HM. Cardiac metastasis from colorectal cancer: a case report. World J Gastroenterol. 2009;15(21):2675-8.

10 Pizzicannella J, Ricci V, Gorla R, Spinapolice E, Esposito A. Isolated cardiac metastasis from colorectal cancer in a 35-year-old man. Case Rep Med. 2012;2012:751761.

11 Chiles C, Woodard PK, Gutierrez FR, Link KM. Metastatic involvement of the heart and pericardium: CT and MR imaging. Radiographics. 2001;21(2):439-49.

12 O'Donnell DH, Abbara S, Chaithiraphan V, Yared K, Killeen RP, Cury RC, et al. Cardiac tumors: optimal cardiac MR sequences and spectrum of imaging appearances. AJR Am J Roentgenol. 2009;193(2):377-87.

13 Murphy MC, Sweeney MS, Putnam JB, Walker WE, Frazier OH, Ott DA, et al. Surgical treatment of cardiac tumors: a 25-year experience. Ann Thorac Surg. 1990;49(4):612-8.

14 Parravicini R, Fahim NA, Cocconcelli F, Barchetti M, Nafeh M, Benassi A, et al. Cardiac metastasis of rectal adenocarcinoma: surgical treatment. Tex Heart Inst J. 1993;20(4):296-8.

15 Tsujii Y, Hayashi Y, Maekawa A, Fujinaga T, Nagai K, Yoshii S, et al. Cardiac metastasis from colon cancer effectively treated with 5-fluorouracil, leucovorin, and oxaliplatin (modified FOLFOX6) plus panitumumab: a case report. BMC Cancer. 2017;17(1):152. 